\title{
Catalase And Peroxiredoxin 5 Protect Xenopus Embryos Against Alcohol-induced Ocular Anomalies
}

M. C. Lin; Institute of Molecular Biology, The University of Hong Kong, Pokfulam, Hong Kong Special

Administrative Region of China

Presentation Number: 2279

Poster Board Number: B7

OBJECTIVES: To study the molecular mechanisms underlying alcohol-induced ocular anomalies in Xenopus embryos. METHODS: Xenopus embryos were exposed to various concentrations (0.1\%-0.5\%) of alcohol and the subsequent effects in eye development and in eye marker gene expression were determined. To investigate the role of reactive oxygen species (ROS) and reactive nitrogen species (RNS) in FAS-associated ocular injury, we overexpressed two anti-oxidant enzymes, catalase and peroxiredoxin 5, in the two blastomeres of the 2-cell stage Xenopus embryos. RESULTS: Exposure of Xenopus embryos to alcohol during eye development produced marked gross ocular anomalies including microphthalmia, incomplete closure of the choroid fissure and malformation of the retina in $40 \%$ of the eyes we examined. In parallel, alcohol (0.1\%-0.5\%) dose-dependently and significantly reduced the expression of several eye marker genes, of which TBX5, VAX2, and Pax6 were the most vulnerable. Over-expression of anti-oxidative enzymes (catalase, cytosolic/peroxisomal and mitochondrial peroxiredoxin 5) significantly decreased the frequency of ocular malformation from $39 \%$ to $21 \%$, $19 \%$, and $13 \%$ respectively. All these enzymes were capable of reducing alcohol-induced ROS production, but only peroxiredoxin 5 could inhibit RNS formation in the alcohol-treated embryos. CONCLUSIONS: The marked protective effects of catalase and peroxiredoxin, particularly the mitochondrial peroxiredoxin 5, suggest for the first time that oxidative stress and nitrosative stress both contribute to alcohol-induced fetal ocular injury.

Key words: Alcohol, Ocular injury, Oxidative stress, Nirosative stress. 